\title{
Qualitative analysis of the minimum flow rate of a cone-jet of a very polar liquid
}

\author{
F. J. Higuera $\dagger$ \\ ETSIAE, Universidad Politécnica de Madrid, Plaza Cardenal Cisneros 3, 28040 Madrid, Spain
}

(Received 30 August 2016: revised 13 February 2017: accepted 14 February 2017)

\begin{abstract}
Electrostatic atomization of a liquid of finite electrical conductivity in the so-called cone-jet regime relies on the electric shear stresses that appear in a region of the liquid surface when a meniscus of the liquid is subjected to an intense electric field. An order of magnitude analysis is used to describe the flow induced by these stresses, which drive the liquid of the meniscus into a jet that issues from the tip of the meniscus and breaks into droplets at some distance from it. When the dielectric constant of the liquid is large, the electric shear stresses extend into the jet and cause a depression that sucks liquid from the meniscus. The induced flow rate is estimated and shown to represent approximately the minimum flow rate at which a cone-jet can be established. It is argued that the meniscus can be stabilized by the electric field that the charge of the jet induces on it. This stabilizing mechanism weakens when the flow rate supplied to the meniscus decreases, and its failure may determine an alternative minimum flow rate for the cone-jet regime. The instability of the jet and existing scaling laws for the size of the spray droplets are discussed.
\end{abstract}

Key words: drops and bubbles, electrohydrodynamic effects

\section{Introduction}

The electrospray is a technique to atomize electrically conductive liquids using electric stresses to overcome the surface tension. In the simplest configuration, a constant flow rate of the liquid to be atomized is injected through a metallic capillary tube into a gas or a vacuum, and a high voltage is applied between this tube and another electrode. In the so-called cone-jet regime, attained in a certain range of voltages and flow rates, the meniscus at the end of the tube takes a conical shape with a thin jet issuing from its tip and breaking into electrically charged droplets at some distance from the meniscus. The radii of the jet and the droplets decrease when the flow rate is decreased or the electric conductivity of the liquid is increased, and they may be very small compared to the radius of the capillary.

The voltage applied to the tube induces electric fields $\boldsymbol{E}=-\nabla \varphi$ and $\boldsymbol{E}^{l}=-\nabla \varphi^{l}$ around the meniscus and in the liquid, respectively, where $\varphi$ and $\varphi^{l}$ are the corresponding electric potentials. Around the meniscus $\nabla^{2} \varphi=0$ in the absence of space charge. In the liquid, the electric field $\boldsymbol{E}^{t}$ originates a conduction current 
density $\boldsymbol{j}=K \boldsymbol{E}^{l}$, where $K$ is the electrical conductivity of the liquid. In the absence of net charge in the bulk of the liquid, $\nabla \cdot j=0$, which amounts to $\nabla^{2} \varphi^{l}=0$. Since the liquid is an electric conductor and the surrounding medium is an insulator, the electric current accumulates free charge at the surface of the liquid. At this surface, the electric field satisfies (Landau \& Lifshitz 1960)

$$
\epsilon_{0}\left(E_{n}-\epsilon E_{n}^{l}\right)=\sigma \quad \text { and } \quad E_{t}=E_{t}^{l} \text {, }
$$

where $\epsilon_{0}$ is the permittivity of the surrounding insulator, $\epsilon$ is the dielectric constant of the liquid, $\sigma$ is the surface density of free charge accumulated by electric conduction and $E_{n}$ and $E_{t}$ are the components of the electric field normal and tangent to the surface. The action of the electric field on the free surface charge and on the charge due to the polarization of the liquid molecules causes a net electric stress (the difference of the electric stresses exerted on the surface by the outer medium and the liquid) whose components normal and tangent to the surface are (Landau \& Lifshitz 1960; Saville 1997)

$$
\tau_{n}^{e}=\frac{1}{2} \epsilon_{0}\left(E_{n}^{2}-\epsilon E_{n}^{l 2}\right)+\frac{1}{2} \epsilon_{0}(\epsilon-1) E_{t}^{2} \quad \text { and } \quad \tau_{t}^{e}=\sigma E_{t} .
$$

The surface charge density satisfies the conservation equation $\partial \sigma / \partial t+v \cdot \nabla \sigma=K E_{n}^{l}-$ $\sigma S$, where $\boldsymbol{v}$ is the velocity of the liquid and $S=-\boldsymbol{n} \cdot \nabla \boldsymbol{v} \cdot \boldsymbol{n}$, with $\boldsymbol{n}$ the unit normal to the surface, is the straining rate of a material element of the surface (Batchelor 1967). Leaving aside for a moment the motion of the liquid, the rate at which conduction brings charge to the surface may be expressed as $(\partial \sigma / \partial t)_{\text {cond }}=K E_{n}^{l}=\left(K / \epsilon_{0} \epsilon\right)\left(\epsilon_{0} E_{n}-\right.$ $\sigma)$, where the first equation (1.1) has been used to write the last equality in terms of the outer field $E_{n}$. For a given value of this field, the free surface charge tends to its equilibrium value $\sigma_{e q}=\epsilon_{0} E_{n}$ in a characteristic time $t_{e}=\epsilon_{0} \epsilon / K$ that depends only on the physical properties of the liquid. This is the electric relaxation time. When it is short compared to the characteristic time of the flow, the free surface charge attains its local equilibrium value, for which the normal field at the liquid side of the surface is zero. The equilibrium surface charge shields the liquid from the outer field.

The velocity and pressure of the liquid satisfy the Navier-Stokes equations. The boundary conditions that express the balance of stresses at the free surface include the electric stress (1.2).

The electric stress tends to elongate the meniscus in the direction of the electric field. It is opposed by the surface tension stress, and a hydrostatic balance can be established in a large part of the meniscus. Equilibrium shapes have been computed by a number of authors for different meniscus configurations leaving out the motion of the liquid altogether (Taylor 1964; Miksis 1981; Wohlhuter \& Basaran 1992). These results show that the response curve giving the elongation of the meniscus as a function of the applied electric field is crescent shaped. No hydrostatic equilibrium is possible for values of the applied field above a critical value that depends on the configuration and marks a turning point of the response curve, while two branches of solutions with different elongations exist in a range of values of the field below the critical value. The solution in the lower branch has an elongation that increases with the applied field and is linearly stable, becoming neutrally stable at the turning point (a generic result; see Iooss \& Joseph 1980). The solution in the upper branch has an elongation that increases when the applied field decreases and is unstable (see, e.g. Wohlhuter \& Basaran 1992). In both branches, any perturbation that increases the elongation of the meniscus (keeping the applied field constant) would increase both the electric stress, because the electric field around the tip intensifies as the tip sharpens, and the surface tension stress, because the curvature of the surface increases. In the upper branch, the electric stress increases more than the surface tension stress, and therefore the perturbation grows. 
The upper branch of unstable solutions ends at a well-defined point where the elongation is maximum and the tip of the meniscus becomes conical. A conical hydrostatic meniscus with a semiangle $\alpha=49.29^{\circ}$ has been found by Taylor (1964) by expressing the balance of surface tension and electric stresses at the equipotential surface of a cone. The electric field around a Taylor cone is of the form $E \sim\left(\gamma / \epsilon_{0} R\right)^{1 / 2}$, where $\gamma$ is the surface tension of the liquid and $R$ is the distance to the vertex. End point solutions featuring a conical tip have been computed by Pantano, Gañán-Calvo \& Barrero (1994) for a meniscus at the end of a capillary tube.

Experimentally, when the voltage applied to the capillary tube of an electrospray is raised, the meniscus is observed to jump from a rounded shape to a conical shape that is remarkably stable. At first sight it is paradoxical that an unstable hydrostatic solution be at the base of a real, stable meniscus. However, as pointed out by Fernández de la Mora (2007), the real meniscus may be stationary but is not hydrostatic. In the cone-jet regime, the charged jet emitted by the tip and the charged droplets into which the jet breaks induce a field on the meniscus that apparently stabilizes it, although it is not yet clear how and when this stabilization occurs.

For a given liquid, a minimum flow rate is known to exist below which a cone-jet cannot be established. Determination of this minimum flow rate is of interest because monodisperse sprays of fine droplets are generated by operating close to it. Estimates of the minimum flow rate as a function of the properties of the liquid have been put forward by Fernández de la Mora \& Loscertales (1994) and Gañán-Calvo, Dávila \& Barrero (1997), in the form

$$
\left(Q_{m i n}\right)_{F L} \sim \frac{\epsilon_{0} \epsilon \gamma}{\rho K} \text { and }\left(Q_{\text {min }}\right)_{G D B} \sim(\epsilon-1)^{1 / 2} \frac{\epsilon_{0} \gamma}{\rho K},
$$

respectively, where $Q$ is the flow rate and $\rho$ is the density of the liquid. The two estimates are not very different, but they are based on fairly different hypotheses on the structure of the flow and the electric field. More recently, Gañan-Calvo, RebolloMuñoz \& Montanero (2013) have proposed that the estimate $\left(Q_{m i n}\right)_{F L}$ should be used when the parameter $R e$ defined in (2.7) below is larger than unity, and should be divided by $R e$ when this parameter is smaller than unity.

These authors and others have proposed scaling laws for the electric current carried by the spray and the size of the droplets in the cone-jet regime as functions of the flow rate and the physical properties of the liquid. The scaling laws of Fernández de la Mora \& Loscertales (1994) are

$$
I_{F L}=f(\epsilon)(\gamma K Q)^{1 / 2} \text { and } D_{d_{F L}}=g(\epsilon)\left(\frac{\epsilon_{0} Q}{K}\right)^{1 / 3},
$$

where $I$ is the current, $D_{d}$ is the diameter of the droplets and $f(\epsilon)$ and $g(\epsilon)$ are empirically determined functions. Those of Gañán-Calvo et al. (1997) have been revised, extended and tested against different experimental results by Gañán-Calvo (1997, 1999, 2004). For values of the flow rate large compared to the minimum, Gañán-Calvo (1999) proposed

$$
I_{G}=2.6(\gamma K Q)^{1 / 2} \quad \text { and } \quad D_{d_{G}}=1.35\left(\frac{\epsilon_{0} \rho Q^{3}}{\gamma K}\right)^{1 / 6} .
$$

Gañán-Calvo (2004) explored the parameter space of the system and found four main scalings and two marginal scalings in as many different regions of this space, whose 
boundaries he identified in terms of dominant balances in the momentum equation for the jet and the relative importance of the two terms of the electric stress $\tau_{n}^{e}$ in (1.2). The reader is referred to the original paper for details.

In both proposals (1.4) and (1.5) the electric current increases as the square root of the product of the flow rate and the conductivity of the liquid, which is in good agreement with experiments and generally accepted in the electrospray literature. The agreement is not as good regarding the size of the droplets, which is predicted to increase with different powers of the flow rate. However, the two predictions give very similar results, to the extent that many experiments have not been able to resolve the difference between them (Rosell-Llompart \& Fernández de la Mora 1994; Chen \& Pui 1997; Gamero-Castaño \& Hruby 2002). More recently, Gañán-Calvo \& Montanero (2009) have obtained strong experimental support for the square root scaling (1.5) for the size of the droplets by gathering data from different authors.

Some of these results are revisited in what follows using order of magnitude analysis to describe the structure of the flow.

\section{Qualitative analysis and results}

The order of the minimum flow rate at which a cone-jet can be established is estimated in $\S \S 2.1$ and 2.2 for values of the dielectric constant of order unity and large, respectively, and some results for larger flow rates are summarized and discussed in $\$ 2.3$.

In the cone-jet regime, leaving aside for the moment stability issues, the electric shear stress sets the liquid in motion and drives it towards the jet. Analysis of the flow induced by the electric shear stress involves various regions around the tip of the conical meniscus and in the jet, including the region where the meniscus departs from a cone and the region where the electric shear stress attains its maximum and the electric current carried by the jet is determined. This flow can be analysed separately from the feeding system that supplies liquid to the meniscus and from the configuration of the electrodes when all these regions are small compared to the size of the meniscus. This is taken to be the case in what follows. The condition is typically satisfied for liquids of moderate and high conductivity at small flow rates, and can be checked a posteriori using the results of the analysis. The flow in these regions is axisymmetric. The meniscus is approximately hydrostatic away from its tip because the hydrodynamic stresses decrease with distance to the tip faster than the normal electric and surface tension stresses. Changing the feeding conditions amounts to changing the pressure in the hydrostatic region. This will change the shape and volume of the meniscus, but the electric field around the tip is not expected to change much as far as the meniscus locally resembles a Taylor cone.

\subsection{Apolar liquids}

The electric shear stress is the product of the surface charge density and the component of the electric field tangent to the surface. In the meniscus, at the small flow rates of interest here, a significant tangent field may exist in a region around its tip where the residence time of the flow is of the order of the electric relaxation time. In the rest of the meniscus the surface charge is nearly at its equilibrium value, and the surface is nearly equipotential. The three terms of the first electrostatic condition (1.1) are of the same order in the relaxation region and, in addition, the components of the electric field in the liquid normal and tangent to the surface are of the same order because the relaxation region is not slender. Thus $E_{t} \sim E_{n}^{l} \sim E_{n} / \epsilon$, and the electric shear stress in the relaxation region is $\tau_{t}^{e}=\sigma E_{t} \sim\left(\epsilon_{0} E_{n}\right)\left(E_{n} / \epsilon\right)$ with $E_{n} \sim\left(\gamma / \epsilon_{0} R_{e}\right)^{1 / 2}$, a subscript $e$ denoting conditions in the relaxation region hereafter. 
The size of the relaxation region and the velocity of the liquid in it, $R_{e}$ and $v_{e}$, can be estimated from the conditions that (i) the residence time is of the order of the electric relaxation time (Fenández de la Mora \& Loscertales 1994), and (ii) the electric shear stress should be balanced either by a viscous shear stress or by the stress due to the inertia of the liquid, whichever is larger; namely $\tau_{t}^{e} \sim \mu v_{e} / R_{e}$ for a viscositydominated flow, where $\mu$ is the viscosity of the liquid, and, approximately, $\tau_{t}^{e} \sim \rho v_{e}^{2}$ for a flow with inertial effects. These conditions give

$$
\left.\begin{array}{c}
R_{e} \sim \frac{\epsilon_{0} \gamma}{\mu K} \quad \text { and } v_{e} \sim \frac{\gamma}{\epsilon \mu}, \\
\text { whence } Q_{e} \sim v_{e} R_{e}^{2} \sim \frac{\epsilon_{0}^{2} \gamma^{3}}{\epsilon \mu^{3} K^{2}} \quad \text { and } \quad I_{e} \sim \sigma v_{e} R_{e} \sim K E_{x}^{l} R_{e}^{2} \sim \frac{\epsilon_{0} \gamma^{2}}{\epsilon \mu^{3 / 2} K^{1 / 2}},
\end{array}\right\}
$$

for a viscosity-dominated flow (small values of $\rho v_{e} R_{e} / \mu \sim \epsilon_{0} \rho \gamma^{2} / \epsilon \mu^{3} K$ ), where $Q_{e}$ and $I_{e}$ are the flow rate and the electric current of the induced flow, and

$$
\left.\begin{array}{c}
R_{e} \sim\left(\frac{\epsilon_{0}^{2} \epsilon \gamma}{\rho K^{2}}\right)^{1 / 3} \text { and } v_{e} \sim\left(\frac{\gamma K}{\epsilon_{0} \epsilon^{2} \rho}\right)^{1 / 3}, \\
\text { whence } Q_{e} \sim \frac{\epsilon_{0} \gamma}{\rho K} \text { and } I_{e} \sim \frac{\epsilon_{0}^{1 / 2} \gamma}{\epsilon^{1 / 2} \rho^{1 / 2}} .
\end{array}\right\}
$$

for a flow with inertial effects $\left(\rho v_{e} R_{e} / \mu \sim \epsilon_{0}^{1 / 3} \rho^{1 / 3} \gamma^{2 / 3} / \epsilon^{1 / 3} \mu K^{1 / 3}\right.$ of order unity or large).

The electric current crossing any section normal to the symmetry axis is made of the contribution of conduction in the bulk of the liquid and convection of the free electric charge accumulated at its surface. The sum of the two contributions is a constant independent of the section considered. Conduction dominates in the meniscus upstream of the relaxation region and convection dominates in the jet downstream, while the two contributions are of the same order in the relaxation region.

The estimation (2.2) of the flow rate for cases with important inertial effects does not depend on the viscosity of the liquid and coincides with the proposed estimations (1.3) of the minimum flow rate up to a factor that depends only on the dielectric constant of the liquid $\epsilon$. However, this result is valid only when the Reynolds number of the flow in the relaxation region is not small. When it is small, as it is for some liquids of interest, the altemative estimation in (2.1) should be used, which is less well supported experimentally.

The hydrodynamic stresses exerted by the induced flow on the surface of the liquid should be of the order of the normal electric and surface tension stresses in order to deform the conical surface and generate a jet. However, the results of this section suggest that this is not possible for large values of $\epsilon$, because the electric shear stress, and thus the pressure and viscous stresses of the induced flow, are smaller than the normal electric stress $\tau_{n}^{e}=O\left(\epsilon_{0} E_{n}^{2}\right)$ by a factor $\epsilon^{-1}$, see the estimation of $\tau_{f}^{e}$ in the first paragraph of this section. The foregoing analysis may be valid for apolar liquids but cannot be correct for polar ones.

\subsection{Polar liquids}

For polar liquids, with large values of the dielectric constant, the electric shear stress drives the liquid in a leading region of the jet rather than in the meniscus 
(Fernández de la Mora \& Loscertales 1994; Gañán-Calvo et al. 1997), and the estimates above must be revised.

Let $x_{e}$ and $r_{s_{e}}$ denote the characteristic length of the region where the electric shear stress comes into play and the characteristic radius of the jet in it. An electric field tangent to the surface and some free surface charge must be present in this region. The conditions for each of these to exist are discussed in turn.

The jet is immersed in the electric field induced by the conical meniscus, which is axial, of the form $E_{x} \sim\left(\gamma / \epsilon_{0} x\right)^{1 / 2}$ in terms of the distance $x$ to the apparent vertex of the cone. This axial field is at first kept out of the liquid by the polarization response it elicits, but it eventually penetrates the liquid and the surface ceases to be equipotential. In the region where this occurs, the radial electric field in the liquid is $E_{r}^{l} \sim\left(r_{s_{e}} / x_{e}\right) E_{x}$, from the condition that the divergence of the electric field is zero. This radial field originates a normal field $E_{r} \sim \epsilon E_{r}$ at the outer side of the surface (see the first equation (1.1)), so that the jet acts as a line of charge and induces an axial field $E_{x_{i n d}} \sim\left(r_{s_{e}} / x_{e}\right) E_{n} \sim \epsilon\left(r_{s_{e}} / x_{e}\right)^{2} E_{x}$ at a distance $r_{s_{e}}$ from the axis (Hinch 1991). The condition that this field be of the order of the field of the conical meniscus determines the characteristic aspect ratio of the region of the jet where the polarization of the liquid can no longer prevent the field of the cone from penetrating the liquid as

$$
\frac{x_{e}}{r_{s_{e}}} \sim \epsilon^{1 / 2} \gg 1 \text {. }
$$

In addition, the residence time of the flow in this region must be of the order of the electric relaxation time $\left(x_{e} / v_{e} \sim \epsilon_{0} \epsilon / K\right)$ in order to have significant levels of surface charge $\left(\sigma_{e} \sim \epsilon_{0} E_{n}\right)$ and electric shear stress $\left(\tau_{t}^{e} \sim \epsilon_{0} E_{n} E_{x}\right)$.

The momentum equation can be integrated across the section of a stationary jet to give, upon using the balance of stresses at the surface (Feng 2002),

$$
\frac{\partial}{\partial x}\left(\pi r_{s}^{2} \rho v^{2}\right)=\frac{\partial}{\partial x}\left(3 \pi r_{s}^{2} \mu \frac{\partial v}{\partial x}\right)+\pi r_{s}^{2} \frac{\partial}{\partial x}\left(\tau_{n}^{e}-\frac{\gamma}{r_{s}}\right)+2 \pi r_{s} \tau_{t}^{e},
$$

where $v$ is a cross-section averaged velocity. In the region of interest, the balance of the tangent electric force (last term of the equation) and either the viscous force or the inertia of the liquid reads $r_{s_{e}}^{2} \mu v_{e} / x_{e}^{2} \sim r_{s_{e}} \tau_{t}^{e}$ for a viscosity-dominated flow and $r_{s_{e}}^{2} \rho v_{e}^{2} / x_{e} \sim r_{s_{e}} \tau_{t}^{e}$ for a flow with inertial effects.

The appropriate form of the balance of forces, together with condition (2.3) and the condition that the residence time is of the order of the electric relaxation time determine the characteristic length $x_{e}$ and the jet radius and velocity, $r_{s_{e}}$ and $v_{e}$, as well as the flow rate and the electric current, as

$$
\left.\begin{array}{c}
x_{e} \sim \frac{\epsilon_{0} \epsilon^{2} \gamma}{\mu K}, \quad r_{s_{e}} \sim \frac{\epsilon_{0} \epsilon^{3 / 2} \gamma}{\mu K}, \quad v_{e} \sim \frac{\epsilon \gamma}{\mu}, \\
Q_{e} \sim v_{e} r_{s_{e}}^{2} \sim \frac{\epsilon_{0}^{2} \epsilon^{4} \gamma^{3}}{\mu^{3} K^{2}}, \quad I_{e} \sim \sigma_{e} v_{e} r_{s_{e}} \sim K E_{x} r_{s_{e}}^{2} \sim \frac{\epsilon_{0} \epsilon^{2} \gamma^{2}}{\mu^{3 / 2} K^{1 / 2}}
\end{array}\right\}
$$

for $\rho v_{e} x_{e} / \mu \sim \epsilon_{0} \epsilon^{3} \rho \gamma^{2} / \mu^{3} K$ small, and

$$
\left.\begin{array}{c}
x_{e} \sim \epsilon\left(\frac{\epsilon_{0}^{2} \gamma}{\rho K^{2}}\right)^{1 / 3}, \quad r_{s_{e}} \sim \epsilon^{1 / 2}\left(\frac{\epsilon_{0}^{2} \gamma}{\rho K^{2}}\right)^{1 / 3}, \quad v_{e} \sim\left(\frac{\gamma K}{\epsilon_{0} \rho}\right)^{1 / 3}, \\
Q_{e} \sim \frac{\epsilon_{0} \epsilon \gamma}{\rho K}, \quad I_{e} \sim \frac{\epsilon_{0}^{1 / 2} \epsilon^{1 / 2} \gamma}{\rho^{1 / 2}}
\end{array}\right\}
$$


for

$$
R e=\left(\frac{\epsilon_{0} \epsilon^{3} \rho \gamma^{2}}{\mu^{3} K}\right)^{1 / 3}
$$

of order unity or large.

The conduction and convection contributions to the electric current are of the same order in the region where (2.5) or (2.6) hold, which is also the current transfer region where the bulk conduction current becomes a surface convection current.

It may be noted in passing that the momentum balance used to obtain (2.6) is equivalent to the energy balance used by Gamero-Castaño \& Hruby (2002) and Gañan-Calvo et al. (2013), according to which the flux of kinetic energy gained by the liquid is of the order of the electric power consumed. Upon multiplying by $x_{e} v_{e}$, the momentum balance reads $\rho v_{e}^{3} r_{s_{e}}^{2} \sim x_{e} \sigma_{e} v_{e} r_{s_{e}} E_{x}$. The left-hand side of this expression is of the order of the flux of kinetic energy across a section of the jet and, since $I_{e} \sim \sigma_{e} v_{e} r_{s_{c}} \sim K E_{x} r_{s_{e}}^{2}$ in the current transfer region, the right-hand side can be written as $I_{e} \Delta \varphi$, where $\Delta \varphi \sim x_{e} E_{x}$ is the electric potential drop in this region. See the supplementary online material of Gamero-Castaño (2010) for a rigorous derivation.

These estimations are for the jet. In the meniscus, the flow is induced by the depression created by the electric suction of the jet. The velocity of this flow is $v_{m} \sim Q_{e} / R^{2}$ in terms of the distance $R$ to the apparent tip. The flow in the meniscus induces pressure and viscous stresses on the surface that become of the order of the surface tension and normal electric stresses, which are the dominating stresses in a conical meniscus, in a certain cone-to-jet transition region where the surface departs from a cone and becomes a jet.

There are a number of possibilities to consider, depending on the conditions of the flow in the current transfer region of the jet and on whether the inertia or the viscosity of the liquid dominates the flow in the meniscus. If viscous forces dominate in the jet, then they also dominate in the meniscus. The balance of normal viscous and surface tension stresses in the cone-to-jet transition region (subscript $t$ hereafter) is $\mu v_{m} / R_{t} \sim$ $\gamma / R_{t}$ and gives, upon using $(2.5)$,

$$
R_{t} \sim \frac{\epsilon_{0} \epsilon^{2} \gamma}{\mu K} \text { and thus } \frac{R_{t}}{v_{m}} \gg \frac{\epsilon_{0} \epsilon}{K} \text { if } R e \ll 1 \text {. }
$$

This $R_{t}$ coincides with $x_{e}$ in $(2.5)$, while the radius of the jet exponentially decreases from $O\left(R_{t}\right)$ in the cone-to-jet transition region to $O\left(r_{s_{e}}\right)$ given by $(2.5)$ in the current transfer region.

If inertial effects are important in the jet, then two possibilities arise for the meniscus. Viscous effect may dominate, in which case $\mu v_{m} / R_{t} \sim \gamma / R_{t}$, or inertial effects may dominate, in which case $\rho v_{m}^{2} \sim \gamma / R_{t}$. Upon using (2.6), these balances give, respectively,

$$
\begin{gathered}
R_{t} \sim\left(\frac{\epsilon_{0} \epsilon \mu}{\rho K}\right)^{1 / 2} \text { and thus } \frac{R_{t}}{v_{m}} \gg \frac{\epsilon_{0} \epsilon}{K} \text { if } 1 \ll R e \ll \epsilon^{2 / 3}, \\
R_{t} \sim\left(\frac{\epsilon_{0}^{2} \epsilon^{2} \gamma}{\rho K^{2}}\right)^{1 / 3} \text { and thus } \frac{R_{t}}{v_{m}} \sim \frac{\epsilon_{0} \epsilon}{K} \text { if } R e \gg \epsilon^{2 / 3} .
\end{gathered}
$$

Table 1 summarizes values of the Reynolds number in the current transfer region of the jet $\left(R e\right.$ in $(2.7)$ ) and in the cone-to-jet transition region $\left(\mathrm{Re} / \epsilon^{2 / 3}\right)$ for various liquids of interest in the ranges of electrical conductivity achieved in 


$\begin{array}{lcccc}\text { Liquid } & \text { Formamide } & \text { Water } & \text { Ethylene glycol } & \text { Triethylene glycol } \\ \epsilon & 111 & 80.1 & 38.66 & 23.69 \\ K\left(\mathrm{~S} \mathrm{~m}^{-1}\right) & 0.028-0.27 & 0.002-0.83 & 5.4 \times 10^{-5}-0.24 & 0.0044-0.045 \\ \operatorname{Re} & 28.64-13.46 & 228.94-30.69 & 13.86-0.84 & 0.80-0.37 \\ \operatorname{Re} / \epsilon^{2 / 3} & 1.24-0.58 & 12.32-1.65 & 1.21-0.074 & 0.097-0.043\end{array}$

TABLE 1. Reynolds numbers for several liquids and conductivity ranges.

the experiments of Fernández de la Mora \& Loscertales (1994). As can be seen, the Reynolds number in the current transfer region is never very large, but inertial effects should be important except for very viscous liquids and large conductivities. The Reynolds number in the transition region is smaller than in the transfer region, and there are cases when viscous effects are small in the transfer region, and the viscosity-independent estimation of the flow rate in (2.6) applies, while viscous forces are still important in the transition region.

The estimations (2.5) and (2.6) give the order of the flow rate that the electric shear force can induce. A stationary solution will be obtained if an identical flow rate is injected through the capillary tube, provided the meniscus is stable at these small flow rates. However, the action of the electric shear stress does not depend on the conditions in the meniscus upstream; it depends only on the electric field acting on the jet and the physical properties of the liquid. If the flow rate injected through the tube is small compared to $Q_{e}$, the electric shear stress will still try to suck a flow of order $Q_{e}$ from the meniscus, decreasing the pressure and probably the volume of the meniscus until no cone can be formed and the emission stops. This suggests that $Q_{e}$ should be about the minimum flow rate for which a cone-jet can be established, if stability constraints do not impose a higher limit.

The estimation of the minimum flow rate in (2.6) for values of $R e$ of order unity or large coincides with the estimation of Fernández de la Mora \& Loscertales (1994), and all the results (2.6) coincide with those of Gañán-Calvo et al. (2013) for their polarization-dominated case. These authors also propose a second lower bound of the flow rate, given by $Q_{e}^{\prime}=\left(\epsilon_{0}^{2} \gamma \mu^{3} / \rho^{4} K^{2}\right)^{1 / 3}$ and based on the condition that viscous forces should never dominate in the current transfer region. Since $Q_{e}$ is large compared to $Q_{e}$ when $R e$ is small $\left(Q_{e}^{\prime} \sim Q_{e} / R e^{4}\right.$ with $Q_{e}$ given by (2.5)), this result implies that the flow rate in (2.5) could never be attained. Gañán-Calvo et al. (2013) derive their result from the assumption that viscous forces at the jet inception prevent the jet from being formed when their effect becomes comparable to the effect of the inertia. However, it is not clear under which conditions should this assumption hold. On the one hand, the result (2.9) implies that viscous forces dominate in a leading region of the jet that does not extend to the current transfer region when $1 \ll R e \ll \epsilon^{2 / 3}$, so that the asumption of Gañán-Calvo et al. (2013) implies that the minimum flow rate in (2.6) could also not be attained in this range of Reynolds numbers, even though it is now larger than $Q_{e}^{\prime}$. On the other hand, although very small values of $R e$ are not common, experimental results show that the Reynolds number $R e_{j}=4 \rho Q / \pi \mu d_{j e t}$ based on the flow rate and the diameter of the jet $d_{j e t}$ may be fairly small in some cases $\left(d_{j e t}\right.$ is typically measured downstream of the current transfer region, after the jet has experienced some additional stretching, so that the value of $R e_{j}$ is an upper bound to the Reynolds number in the current transfer region). Thus, the results of Alonso-Matilla et al. (2014) with mixtures of ethylammonium 
nitrate and sulfolane electrosprayed in vacuo give $R e_{j} \approx 0.12$; Hijano \& Loscertales (private communication) find values of $R e_{j}$ of 0.05 or smaller in experiments with glycerol doped with $\mathrm{NaCl}$, while Jayasinghe \& Edirisinghe (2002) report significantly smaller values for the same liquid doped with citric acid; and Loscertales et al. (2002) find $R e_{j} \approx 0.15$ in experiments carried out with $S$ omos 6120 , a photopolymer that acts nearly as a Newtonian liquid in the conditions of their experiments (the measured diameters of single jets of Somos 6120, not reported in their paper, were kindly supplied by Professor I. G. Loscertales). In addition, numerical solutions of the Stokes equations featuring a stationary electrically driven jet have been computed in Higuera (2010).

\subsection{Higher flow rates and stability issues}

A cone-jet can also be established when the flow rate injected through the tube is large compared to $Q_{e}$. Increasing the flow rate increases the speed of the liquid in the meniscus and the convection of surface charge into the jet. In the meniscus, the normal hydrodynamic stress of the flow on the surface becomes of the order of the surface tension and normal electric stresses before relaxation effects come into play. Thus, for a flow with important inertial effects, $\gamma / R \sim \rho v^{2}$ (with $v \sim Q / R^{2}$ ) for $R \sim R_{h}=$ $\rho^{1 / 3} Q^{2 / 3} / \gamma^{1 / 3}$ (Fernández de la Mora et al. 1990), and $\left(R_{h} / v\right) /\left(\epsilon_{0} \epsilon / K\right) \sim Q / Q_{e} \gg 1$ in this cone-to-jet transition region. In the jet, there is plenty of surface charge to induce a normal field at the outer side of the surface without having to rely on the electric displacement of the liquid. Estimates for $Q \gg Q_{e}$ have been worked out elsewhere (Higuera 2003) and will be briefly summarized here for the case when inertial effects are important.

As in $\$ 2.2$, significant electric shear stresses exist only in the region of the jet where the field $E_{x} \sim\left(\gamma / \epsilon_{0} x\right)^{1 / 2}$ induced by the conical meniscus first penetrates the liquid and the surface ceases to the equipotential. Characteristic magnitudes pertaining to this region are denoted with a subscript $c t$ in what follows, which stands for current transfer. In contrast to the case of $\$ 2.2$, the electric field of the cone on the jet is now opposed by the axial field induced by the free surface charge of the jet, rather than by the electric displacement of the liquid. The field of the cone penetrates the jet when the rate at which conduction accumulates charge at its surface does not suffice to keep this balance of axial fields. The radial electric field in the liquid is then $E_{r}^{l} \sim\left(r_{s_{i t}} / x_{c t}\right) E_{x}$, from the condition $\nabla \cdot \boldsymbol{E}^{l}=0$. This field induces a radial conduction current that accumulates charge at the moving material surface of the liquid at a rate $v \mathrm{~d} \sigma / \mathrm{d} x \sim K E_{n}^{l}$, with $v \sim Q / r_{s_{i z}}^{2}$. The surface charge density is therefore $\sigma \sim K E_{r}^{l} x_{c t} / v \sim$ $K r_{s_{i t}}^{3} E_{x} / Q$, which, from (1.1a), advancing that $\epsilon_{0} \epsilon E_{n}^{l} / \sigma \sim\left(\epsilon_{0} \epsilon / K\right) /\left(x_{c r} / \nu\right) \ll 1$, leads to a normal electric field $E_{s} \approx \sigma / \epsilon_{0}$ at the outer side of the surface. As in $\$ 2.2$, the jet acts as a line of charge extending along the symmetry axis, though this is now due to the free surface charge rather than to the polarization of the liquid. The axial field induced by this line of charge at a distance $r_{s_{i f}}$ from the axis is $E_{x_{i, d}} \sim$ $\left(r_{s_{i t}} / x_{c t}\right) E_{n} \sim\left(K r_{s_{c t}}^{4} / \epsilon_{n} x_{c t} Q\right) E_{x}$. Since the tangential field is continuous at the surface of the jet, the condition that $E_{x_{\text {ind }}}$ be of the order of the field due to the charge of the conical meniscus and partially offset it defines the region where this field first penetrates the jet. This condition, together with the balance of electric shear force and liquid inertia (thinking of the case of high Reynolds number flow; see Higuera (2003) for viscosity-dominated flow), determines the characteristic length and radius of the jet in the region of interest,

$$
x_{c t} \sim \frac{\rho^{2 / 3} K^{1 / 3} Q}{\epsilon_{0}^{1 / 3} \gamma^{2 / 3}} \sim R_{h}\left(\frac{\epsilon Q}{Q_{e}}\right)^{1 / 3} \quad \text { and } \quad r_{s_{t \rightarrow}} \sim \frac{\epsilon_{0}^{1 / 6} \rho^{1 / 6} Q^{1 / 2}}{\gamma^{1 / 6} K^{1 / 6}} \sim \frac{R_{h}}{\left(\epsilon Q / Q_{e}\right)^{1 / 6}} .
$$


This region has the following properties: it is slender, $x_{c t} / r_{s c t} \sim\left(\epsilon Q / Q_{e}\right)^{1 / 2} \gg 1$; relaxation effects are negligible, $\left(x_{c t} / v\right) /\left(\epsilon_{0} \epsilon / K\right) \sim Q / Q_{e} \gg 1$, as was anticipated above; the flow-induced depression is balanced in first approximation by a normal electric stress, which is large compared to the surface tension stress, $\rho v_{c t}^{2} \sim \tau_{n}^{e} \gg \gamma / r_{s_{c}}$; and the conduction and convection components of the electric current are of the same order, $I \sim \sigma v_{c t} s_{s_{c t}} \sim K E_{x} r_{s c t}^{2} \sim(\gamma K Q)^{1 / 2}$. The estimated current coincides with the scaling law of Gañán-Calvo et al. (1997) and differs from the scaling law of Fernández de la Mora \& Loscertales (1994) by a factor that is a function of $\epsilon$.

At the surface of the jet upstream of the current transfer region, for $R_{h} \ll x \ll x_{c t}$, (i) the axial field induced by the surface charge almost exactly balances the field of the meniscus $\left(E_{x_{\text {ind }}} \approx-E_{x}\right)$, rendering the surface equipotential, and (ii) the normal electric stress causes a depression that accelerates the liquid. These two conditions lead to $r_{s} \sim \rho^{1 / 2} Q / \gamma^{1 / 2} x^{1 / 2}$ and $E_{n} \sim \gamma x / \epsilon_{0}^{1 / 2} \rho^{1 / 2} Q$.

The electric field induced by the charge of the jet modifies the field on the meniscus at distances of order $x_{c t}$ upstream of the tip. This may have a stabilizing effect. Consider a perturbation that increases the elongation of the meniscus. In the absence of the jet, this perturbation would grow because the meniscus is unstable. However, the perturbation changes the field $E_{x}$ acting on the jet, and thus causes a change of the charge distribution of the jet, which tends to a new stationary state in a time that can be shown to be of the order of the residence time $t_{r}=x_{c t} / v_{c t}$ (Higuera 2008). The field induced by the new charge distribution opposes the change of $E_{x}$ because it has to partially offset it. Since this field acts also on the meniscus, and the jet response time $t_{r}$ is short compared to the capillary time $t_{c}=\left(\rho x_{c t}^{3} / \gamma\right)^{1 / 2}$ in which the perturbation of the meniscus is expected to evolve $\left[t_{c} / t_{r} \sim\left(\epsilon Q / Q_{e}\right)^{1 / 2} \gg 1\right]$, the charged jet has the potential to stop the growth of the perturbation, though a quantitative stability analysis would be needed to assess this possibility.

The length and charge of the current transfer region decrease with the flow rate, and so does the stabilizing effect of this charge. This might determine a new minimum flow rate if the cone-jet becomes unstable at a flow rate larger than $Q_{e}$ estimated in (2.5) or (2.6). In particular, loss of stability would provide an alternative explanation for the minimum flow rates larger than $Q_{e}$ in (2.5) observed in the data gathered by Gañán-Calvo et al. (2013) for $R e<1$. In fact, small values of $R e$ favour this loss of stability because the current transfer region is then shorter than at high $R e$. Thus, the radius of the jet decreases exponentially with streamwise distance in the absence of inertial effects, from values of the order of the size of the cone-to-jet transition region, $R_{v}=(\mu Q / \gamma)^{1 / 2}$, to values of $O\left[R_{v} / \epsilon^{1 / 2}\left(Q / Q_{e}\right)^{1 / 8}\right]$ in the current transfer region, keeping $x=O\left(R_{v}\right)$ (Higuera 2003).

In any case, the stabilizing mechanism described above cannot directly stop the growth of perturbations extending to regions of the meniscus of size large compared to $x_{c t}$, because the field induced by the charge of regions of the jet long compared to $x_{c t}$ is small compared to the Taylor field due to the meniscus. The space charge of the spray of charged droplets, or the finite size of the meniscus, or a pinning effect due to the effective singularity at its tip (Coffman et al. 2016) can play a role here, but again a quantitative analysis is needed.

The estimations (2.5), (2.6) and (2.11) are for a stationary jet, but the jet of an electrospray must eventually break into droplets. The charge of the jet and the electric field into which it is immersed have an effect on its instability (Hohman et al. $2001 a, b$ ). Thus, the stretching due first to the electric-induced depression and then to the electric shear stress seems to stabilize the jet, while the mutual repulsion of the charges it carries originates new instabilities, some of which are not axisymmetric. 
Despite these complexities, the final stages of the jet's evolution are sometimes similar to those of the breakup of an uncharged jet due to Rayleigh instability (Tang \& Gomez 1994; Fernández de la Mora 2007). In particular, the diameter of the droplets may be commensurate with the diameter of the jet at the breakup point.

The estimations worked out above have a bearing on the stability of the jet. Surface tension and normal hydrodynamic stresses acting on the surface are of the same order in the cone-to-jet transition region of characteristic size $R_{h}$. However, if the jet extends far beyond this region and is significantly stretched by the electric-induced depression, as the results (2.11) suggest for large values of $\epsilon Q / Q_{e}$, then the surface tension stress increases less rapidly than the hydrodynamic stress with decreasing jet radius; namely as $\gamma / r_{s}$ versus $\rho v^{2} \sim \rho Q^{2} / r_{s}^{4}$ for a flow with important inertial effects. This leaves only normal electric and hydrodynamic stresses to balance each other in an ample region of the jet (see, e.g. the third paragraph of this section), which is therefore subjected to Frenkel instability (Landau \& Lifshitz 1960). López-Herrera, Riesco-Chueca \& Gañán-Calvo (2005) showed that, in these conditions, an unstretched jet is unstable to perturbations in a wide range of wavenumbers. For a very polar liquid with a planar surface under a uniform electric field $E_{n}$, the growth rate of a small perturbation proportional to $\exp (\mathrm{i} k x+s t)$ due to Frenkel instability is $s=\left(\epsilon_{0} E_{n}^{2}-\gamma k\right) / 2 \mu$ when viscosity dominates the evolution of the perturbation (case $\mathrm{i}$ in what follows), and $s=k\left(\epsilon_{0} E_{n}^{2}-\gamma k\right)^{1 / 2} / \rho^{1 / 2}$ when the inertia dominates (case ii). The surface is unstable to perturbations with wavenumber smaller than $k_{c}=\epsilon_{0} E_{n}^{2} / \gamma$. The maximum growth rate is $s_{\max }=\epsilon_{0} E_{n}^{2} / 2 \mu$, for $k=0$, in case (i), and $s_{\max }=\left(2 / 3^{3 / 2}\right) \epsilon_{0}^{3 / 2} E_{n}^{3} / \rho^{1 / 2} \gamma$, for $k=2 k_{c} / 3$, in case (ii). Since $k_{c} r_{s} \sim\left(\epsilon Q / Q_{e}\right)^{1 / 2}\left(x / x_{c t}\right)^{3 / 2}$ is large in most of the jet, these results for a planar surface are approximately applicable to the jet. In case (ii), the instability should be expected to generate droplets of radius small compared to the radius of the jet, while in case (i) the droplets should have the largest possible radius, which is of $O\left(r_{s}\right)$. Case (i) corresponds to $\mu / r_{s}^{2} \gg \rho s_{\max }$, which amounts to $\epsilon / R e^{2} \gg\left(Q / Q_{e}\right)\left(x / x_{c t}\right)$, a condition that can be realized for small flow rates. In both cases, the growth rate of the instability is large compared to the inverse of the residence time in the current transfer region. These results suggest that the jet can maintain its integrity up to the current transfer region only for moderate values of $\epsilon Q / Q_{e}$, which is in agreement with the observations of Fernández de la Mora et al. (1990) and Chen, Pui \& Kaufman (1995) and with the conclusions of López-Herrera \& Gañán-Calvo (2004). However, the growth rate can be reduced by the stabilizing effect of jet stretching, which has not been accounted for here. In any case, the discussion suggests a number of possibilities for the jet dynamics. The jet needs not always break in the current transfer region through the Rayleigh capillary instability.

The square root law for the size of the spray droplets is obtained if the jet breaks in the current transfer region into droplets of radius $r_{d}$ commensurate with the jet's radius $r_{s_{c t}}$ in (2.11). The experimental results gathered by Gañán-Calvo \& Montanero (2009) show that this law holds in a wide range of flow rates. Using the scalings (1.5) of Gañán-Calvo (1999) for the diameter of the droplets and the electric current as functions of the flow rate, which contain experimentally determined numerical factors, the condition $q_{d} /\left(4 \pi r_{d}^{3} / 3\right)=I / Q$, where $q_{d}$ is the charge of the droplets, gives $q_{d} / q_{R}=$ $0.24\left(\epsilon Q / Q_{e}\right)^{1 / 4}$. Here $Q_{e}=\epsilon_{0} \epsilon \gamma / \rho K$ (cf. (2.6)) and $q_{R}=8 \pi\left(\epsilon_{0} \gamma r_{d}^{3}\right)^{1 / 2}$ is the Rayleigh limit charge (Rayleigh 1882), which is the maximum charge that a stable droplet can bear. The ratio $q_{d} / q_{R}$ is predicted to be large for large values of $\epsilon Q / Q_{e}$, in which case the droplets should undergo Coulomb explosions immediately upon being generated. However, $q_{d} / q_{R}<1$ for $Q / Q_{e}<296.4 / \epsilon$, which nearly coincides with the condition $W e<20$ of Gañán-Calvo \& Montanero (2009) and defines a range of flow rates where 
the droplets are stable. In addition, the conditions of conservation of liquid volume and charge during breakup can be used to write the radius and surface density of charge of the jet at the breakup position, $r_{s_{b}}$ and $\sigma_{b}$ say, in terms of the radius and charge of the droplets and the wavelength $\lambda$ of the perturbation that causes the breakup; namely $\pi r_{s_{b}}^{2} \lambda=4 \pi r_{d}^{3} / 3$ and $2 \pi r_{s_{b}} \lambda \sigma_{b}=q_{d}$. Using these conditions, the ratio of normal electric to surface tension stress at the breakup position of the jet can be evaluated as $\left(\sigma_{b}^{2} / 2 \epsilon_{0}\right) /\left(\gamma / r_{s_{b}}\right)=b\left(q_{d} / q_{R}\right)^{2}$, where $b=6 /\left(\lambda / r_{s_{b}}\right)=0.67$ for $\lambda / r_{s_{b}}=9.01\left(r_{d} / r_{s_{b}}=\right.$ 1.89), corresponding to the most amplified wavelength of Rayleigh capillary instability in the absence of surface charge, and $b=1.59$ for $\lambda / r_{s_{b}}=3.80\left(r_{d} / r_{s_{b}}=1.42\right)$, which is the wavelength leading to the value of $\sigma_{b}$ given by Gañan-Calvo (1999). In summary, the condition of stability of the droplets, $q_{d} / q_{R}<1$, sets an upper bound to the electric to surface tension stress ratio in the jet, which cannot reach the large values assumed in the foregoing estimations.

If the jet is assumed to survive beyond the current transfer region, then the streamwise variation of its radius can be estimated as (Gañán-Calvo 1997)

$$
r_{s} \sim\left(\frac{\epsilon_{0} \rho^{2} Q^{5}}{\gamma^{2} K x}\right)^{1 / 8}
$$

from the balance of liquid inertia and electric shear force, $2 \pi r_{s} \tau_{t}^{e} \sim \partial\left(\pi r_{s}^{2} \rho v^{2}\right) / \partial x$, in regions where the surface charge density is determined by the condition that the electric current is essentially surface convection current, $\sigma \sim I /\left(r_{s} v\right)$, and the electric field acting on the jet is still that of a Taylor cone, $E_{t} \sim\left(\gamma / \epsilon_{0} x\right)^{1 / 2}$. Using these results, the ratio of surface tension to normal electric stress can be seen to increase with streamwise distance, $\left(\gamma / r_{s}\right) / \tau_{n}^{e} \sim\left(Q_{e} / \epsilon Q\right)^{1 / 2}\left(x / x_{c t}\right)^{3 / 8}$, and to become of order unity at distances from the meniscus of order $x_{c}=\rho^{2} K^{5 / 3} Q^{7 / 3} / \epsilon_{0}^{5 / 3} \gamma^{2}$, where the radius of the jet has decreased to values of order $r_{s_{c}}=\left(\epsilon_{0} Q / K\right)^{1 / 3}$. The Rayleigh capillary instability may cause the breakup of the jet in this region, and the diameter of the droplets would then be of the order of the local radius of the jet, in agreement with the cubic root law (1.4b) of Fernández de la Mora \& Loscertales (1994) except for the function $g(\epsilon)$. The charge of the droplets would be of the order of the Rayleigh limit charge, also in agreement with experimental results (Gomez \& Tang 1994; Tang \& Gomez 1994; Chen et al. 1995; de Juan \& Fernández de la Mora 1997; Gañán-Calvo et al. 1997). The viscosity of the liquid will affect the breakup of the jet and the size of the droplets when $\rho \gamma r_{s} / \mu^{2} \sim \epsilon^{-5 / 3} \operatorname{Re}^{2}\left(Q / Q_{e}\right)^{1 / 3}$ is not large. These results hold when $x_{c}$ is small compared to the size of the meniscus. If this condition is not satisfied, then the jet comes into a region where the prevailing electric field is not that of a Taylor cone and depends on the configuration of meniscus and the electrodes. Gañán-Calvo \& Montanero (2009) argue that the square root law for the size of the droplets is obtained in this case when the electric potential drops and the jet acquires a constant kinetic energy. Similarly, the results (2.11) and (2.5) and (2.6) of the previous section require that $x_{c t}$ and $x_{e}$, respectively, be small compared to the size of the meniscus.

\section{Conclusions}

The structure of the flow induced in a cone-jet by the electric shear stress at the surface of the liquid has been described using an order of magnitude analysis. For polar liquids, the electric shear stress acts in a slender region of the jet where electric relaxation effects are important, and generates a depression that sucks liquid from the meniscus across a cone-to-jet transition region. The flow rate and the electric current carried by the jet have been estimated for inertia- and viscosity-dominated flows. It is argued that the estimated flow rate should be of the order of the minimum for which a cone-jet can be established, if stability constraints allow reaching this flow rate. 
Estimates for injected flow rates large compared to this minimum suggest that electric relaxation effects are not important anywhere in the flow. The electric field induced by the charged jet on the meniscus rapidly responds to perturbations of the meniscus and, by opposing them, has the potential to stabilize the meniscus. The instability of the jet and existing scaling laws for the size of the spray droplets have been examined in the light of these estimations.

\section{Acknowledgements}

I an grateful to A. Hijano and I. G. Loscertales (University of Málaga) who kindly provided unpublished data from their experiments. This work was supported through the Spanish MINECO projects DPI2013-47372-C02-02 and DPI2015-71901-REDT.

\section{REFERENCES}

alonso-Matilla, R., Fernández-García, J., Congdon, H. \& Fernández de la Mora, J. 2014 Search for liquids electrospraying the smallest possible nanodrops in vacuo. J. Appl. Phys. 116, 224504.

BatcheloR. G. K. 1967 An Introduction to Fluid Dynamics. Cambridge University Press.

CHEN, D. R. \& PUI, D. Y. H. 1997 Experimental investigation of scaling laws for electrospraying: dielectric constant effects. Aerosol Sci. Technol. 27, 367-380.

Chen, D. R., Pui, D. Y. H. \& Kaufman, S. L. 1995 Electrospraying of conducting liquids for monodisperse aerosol generation in the $4 \mathrm{~nm}$ to $1.8 \mu \mathrm{m}$ diameter range. Aerosol Sci. Technol. 26, 963-977.

Coffman. C., Martinez-Sánchez, M.. Higuera. F. J. \& Lozano, P. C. 2016 Structure of the menisci of leaky dielectric liquids during electrically-assisted evaporation of ions. Appl. Phys. Lett. 109, 231602.

FENG, J, J, 2002 The stretching of an electrified non-Newtonian jet: a model for electrospinning. Phys. Fluids 14. 3912-3926.

FERnÁndeZ DE LA MORA, J. 2007 The fluid dynamics of Taylor cones. Annu. Rev. Fluid Mech. $39,217-243$.

Fernández de la Mora, J. \& Loscertales, I. G. 1994 The current emitted by highly conducting Taylor cones. J. Flaid Mech. 260, 155-184.

Fernández de la Mora, J., Navascues, J., Fernández, F. \& Rosell-Llompart, J, 1990 Generation of submicron monodisperse aerosols in electrosprays. J. Aero. Sci. 21 (Suppl.1), S673-S676.

Gamero-CASTAÑo, M. 2010 Energy dissipation in electrosprays and the geometric scaling of the transition region of cone-jets. J. Fluid Mech. 662, 493-513.

GAMERo-CASTANO, M. \& HRUBY, V. 2002 Electric measurements of charged sprays emitted by cone-jets. J. Fluid Mech. 459, 245-276.

GAÑÁN-CALVO. A. M. 1997 Cone-jet analytical extension of Taylor's electrostatic solution and the asymptotic universal scaling laws in electrospraying. Phys. Rev. Lett. 79. 217-220.

GAÑÁ-CALVO. A. M. 1999 The surface charge in electrostatic spraying: its nature and its universal scaling laws. J. Aero. Sci. 30, 863-872.

GAÑÁN-CALVo, A. M. 2004 On the general scaling theory for electrospraying. J. Fluid Mech. 507, 203-212.

Gañán-Calvo. A. M., Dávila, J. \& Barrero. A. 1997 Curtent and drop size in the electrospraying of liquicls. Scaling laws. J. Aero. Sci. 28. 249-275.

Gañan-Calvo. A. M. \& Montanero, J. M. 2009 Revision of capillary cone-jet physics: electrospray and flow focusing. Phys. Rev. E 79, 066305.

Gañán-Calvo, A. M., Rebollo-Muñoz, N. \& Montanero, J. M. 2013 The minimum or natural rate of flow and droplet size injected by Taylor cone-jets: physical symmetries and scaling laws. New J. Phys. 15. 033035. 
Gomez, A. \& TANG, K. 1994 Charge and fission of droplets in electrostatic sprays. Phys. Fluids 6, $404-414$.

Higuera, F. J. 2003 Flow rate and electric current emitted by a Taylor cone. J. Fluid Mech. 484, 303-327.

HigueRA. F. J. 2008 Breakup of a supported drop of a viscous conducting liquid in a unifom electric field. Phys. Rev. E 78. 016314.

Higuera, F. J. 2010 Numerical computation of the domain of operation of an electrospray of a very viscous liquid. $J$. Fluid Mech. 648, 35-52.

Hinch. E. J. 1991 Perturbation Methods. Cambridge University Press.

Hohman. M. M.. Shin, M. Rutledge, G. \& BRenneR, M. P. $2001 a$ Electrospinning and electrically forced jets. I. Stability. Phys. Fluids 11. 2201-2220.

Hohman. M. M.. Shin, M.. Rutledge, G. \& BRenneR, M. P. $2001 b$ Electrospinning and electrically forced jets. II. Applications. Phys. Fluids 11, 2221-2236.

IOOSS, G. \& JOSEPH. D. D. 1980 Elementary Stability and Bifituation Theory. Springer.

JAYASINGHE, S. N. \& Edirisinghe, M. J. 2002 Effect of viscosity on the size of relics produced by electrostatic atomization. J. Aero. Sci. 33. 1379-1388.

DE JUAN, L. \& FERNÁNDEZ DE LA MORA, J. 1997 Charge and size distributions of electrospray drops. J. Colloid Interface Sci. 186. 280-293.

Landau, L. D. \& Lifshitz, E. M. 1960 Electrodynamics of Continuous Media. Pergamon.

López-HerRera, J. M. \& GAÑÁn-CALvo, A. M. 2004 A note on charged capillary jet breakup of conducting liquids: experimental validation of a viscous one-dimensional model. J. Fluid Mech. 501. 303-326.

López-Herrera, J. M. Riesco-Chueca, P. \& Gañán-Calvo. A. M. 2005 Linear stability analysis of axisymmetric perturbations in imperfectly conducting liquid jets. Phys. Fluids 17, 034106.

Loscertales, I. G., Barrero, A., Guerrero, I., Cortijo, R., Marquez, M. \& Gañán-Calvo, A. M. 2002 Micro/nano encapsulation in electrified coaxial liquid jets. Science 33, 1379-1388.

MiKsIS, M. J. 1981 Shape of a drop in an electric field. Phys. Fluids 24. 1967-1972.

Pantano, C.. GañÁn-Calvo, A. M. \& Barrero, A. 1994 Zeroth-orler, electrolydrostatic solution for electrospraying in cone-jet mode. J. Aero. Sci. 25. 1065-1077.

RAYleigh, LORD 1882 On the equilibrium of conducting masses charged with electricity. Phil. Mag. 14. 184-186.

Rosell-Llompart, J, \& Fernández de LA MORA, J, 1994 Generation of monodisperse droplets $0.3-4 \mu \mathrm{m}$ in diameter from electrified cone-jets of highly conducting viscous liquids. J. Aero. Sci. 25, 1093-1119.

SAVILLE, D. A. 1997 Electrohydrolynamics: the Taylor-Melcher leaky dielectric model. Annu. Rev. Fluid Mech. 29, 27-64.

TANG, K.\& GOMEZ, A. 1994 On the structure of an electrostatic spray of monodisperse droplets. Phys. Fluids 6, 2317-2332.

TAYLoR. G. I. 1964 Disintegration of water drops in an electric field. Proc. $R$. Soc. Lond. A 280, 383-397.

WOHLhUTER. F. K. \& BaSARAN, O. A. 1992 Shapes and stability of pendant and sessile dielectric drops in an electric field. J. Fluid Mech. 235, 481-510. 